\title{
DNA Annealing Helicase and Endonuclease ZRANB3
}

National Cancer Institute

\section{Source}

National Cancer Institute. DNA Annealing Helicase and Endonuclease ZRANB3. NCI

Thesaurus. Code C116087.

DNA annealing helicase and endonuclease ZRANB3 (1079 aa, 123 kDa) is encoded by the human ZRANB3 gene. This protein is involved in both the modification of DNA topology and the response to DNA damage. 